\title{
Iron in fibrous diamonds from different localities: X-ray Absorption and Mössbauer data
}

\author{
Andrei A. Shiryaev ${ }^{1}$, Yan V. Zubavichus ${ }^{2}$, Catherine McCammon ${ }^{3}$, Alexei A. Veligzhanin ${ }^{2}$ \\ ${ }^{1}$ Institute of Crystallography, Moscow, Russia \\ ${ }^{2}$ Russian Research Center "Kurchatov Institute", Moscow, Russia \\ ${ }^{3}$ Bayerisches Geoinstitute, Bayreuth, Germany
}

Fibrous diamonds are an important source of information about fluids in the Earth's upper mantle. Currently, many results obtained for the fibrous crystals are generalised for all diamonds, though fibrous stones are relatively rare and differ in many respects from more abundant crystals grown by the tangential growth mechanism. To check the validity of such extrapolations it is important to perform complementary studies of fibrous diamonds. Up to now no information about the redox conditions of fibrous diamond formation was available. In this study we have attempted to determine the Fe redox state and environment in fibrous diamonds from Zaire and Brazil using X-ray Absorption (XAS) and Mössbauer spectroscopies. It should be noted that each fibrous diamond contain millions of multiphased chemically complex inclusions. Therefore, presented data provide information averaged over numerous inclusions.

\section{Samples and methods.}

Cubic diamonds from Brazilian placers $(n=8)$ and from Zaire (presumably, Mbuji-Maji) $(n=8)$ were studied by X-ray Absorption Spectroscopy (XAS) at the Fe Kedge. All diamonds were characterized by IR spectroscopy. In addition, the most H-rich samples were investigated by ${ }^{1} \mathrm{H}$ Nuclear Magnetic Resonance by Shiryaev et al., (2008). XAS spectra were obtained on severely acid treated whole diamonds in transmission and in fluorescence modes using the synchrotron source at Russian Research Center "Kurchatov Institute"; various iron compounds were employed for standardization. The measurements were performed at room temperature and the main emphasis was on the Near-Edge region, i.e., XANES part of the spectra (usually from $\sim 60 \mathrm{eV}$ below till $\sim 100 \mathrm{eV}$ after the absorption edge). The size of incident X-ray beam was approx. $1 \mathrm{~mm}^{2}$.

Another set of samples consist of four plates laser-cut from Brazilian fibrous and coated diamonds to reveal an internal structure. These diamonds were described in considerable details by Shiryaev et al., (2005). These diamonds are remarkable since chemistry of microinclusions reflects gradual evolution of growth mèdium. Mössbauer measurements of these fibrous diamonds were made of the bulk sample as well as on a few specific regions that showed the greatest contrast on cathodoluminescence images and variations in carbon isotopic composition as reported by Shiryaev et al. (2005). A piece of $25 \mu \mathrm{m}$ thick Ta foil was drilled with a hole and placed over the area to be studied. The diameter of the hole varied from 400 to $1000 \mu \mathrm{m}$, depending on the size of the region to be studied. The thickness of the plates ranged from 380 to $420 \mu \mathrm{m}$, and was used in conjunction with electron microprobe analyses (Shiryaev et al., 2005) to estimate Mössbauer thicknesses to range from 5 to $10 \mathrm{mg} \mathrm{Fe} / \mathrm{cm}^{2}$. The spectra were recorded at room temperature in transmission mode on a constant acceleration. Spectra of bulk samples took 3-4 days each to collect, while spectra of specific regions took 6-13 days each to collect, and all spectra were fitted using the commercially available fitting program NORMOS.

\section{Results}

\section{$X$-ray Absorption Spectroscopy}

The XANES measurements reveal variability between Fe K-edge spectra of different diamonds, clearly pointing to the existence of several iron-containing phases. The position, shape and intensity of the preedge peak in the XANES spectrum can be used for estimation of the $\mathrm{Fe}^{2+} / \mathrm{Fe}^{3+}$ ratio and depends on the symmetry of the Fe environment. The samples form two main groups:

- spectra of 6 Brazilian and 3 Zairean diamonds resemble (but are not identical to!) synthetic $\mathrm{Fe}_{2} \mathrm{O}_{3}$

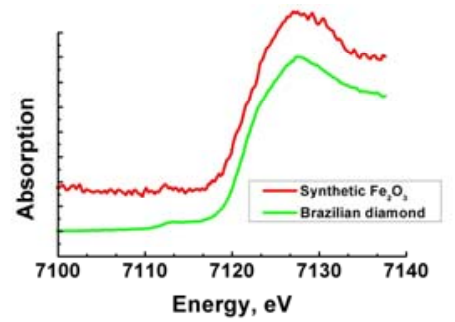

(Fig. 1);

Figure 1. XANES spectra of synthetic $\mathrm{Fe}_{2} \mathrm{O}_{3}$ and one of diamonds from the first group.

- spectra showing very weak pre-edge peak, but intense "white" line (fig. 2). Such spectra could be 
interpreted as $\mathrm{Fe}^{2+}$ present in 8-fold coordination, resembling, e.g., spectrum of almandine (Wilke et al., 2001). 3 Zairean and one Brazilian diamonds belong to this group.

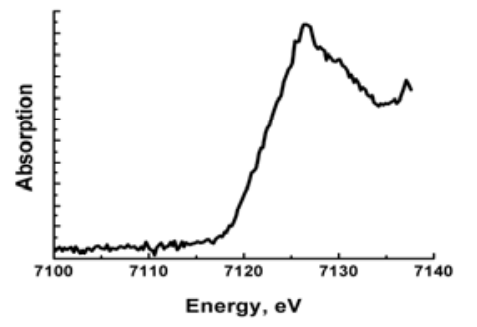

Figure 2. XANES spectrum of a diamond from the second group.

Three other samples show somewhat different spectra; the most pronounced difference being in the amplitude of the pre-edge peak. In one of the samples we may suspect the presence of $10-15 \%$ of metallic Fe (fig. 3). One spectrum is similar to that of $\mathrm{Fe}$ sulphate. It is known that in this compound the $\mathrm{Fe}$ cation is surrounded by water molecules. In the context of mantle-related phases the spectrum of this sample is consistent with the presence of Fe-bearing water-rich melt/glass. Limited data indicate that in the studied samples $\mathrm{Fe}^{3+}$ (when observed) is present mostly in tetrahedral and in octahedral sites.

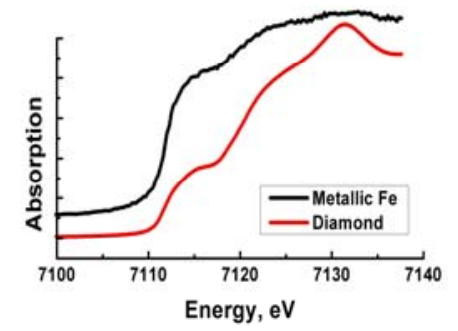

Figure 3. XANES spectrum of metallic $\mathrm{Fe}$ and from one of the diamonds.

\section{Mössbauer spectroscopy}

Mössbauer spectra of all samples are similar (Fig. 4), and show a broad absorption superimposed by a narrow doublet near $0 \mathrm{~mm} / \mathrm{s}$. The spectra were therefore fitted to a Lorentzian quadrupole doublet and a Lorentzian magnetic sextet with centre shift constrained to be equal to that of the quadrupole doublet. The latter constraint was added for consistency between spectra, but did not change the results. In some spectra a second Lorentzian quadrupole doublet was added to account for additional absorption near $+2 \mathrm{~mm} / \mathrm{s}$. Conventional constraints were applied to the components of each doublet (i.e., equal area and width) and sextet.

The centre shifts of the spectral components can be used to infer the nature of iron corresponding to each absorption feature. The centre shift of the narrow quadrupole doublet and magnetic sextet are close to 0 $\mathrm{mm} / \mathrm{s}$, and indicate iron occurring either as $\mathrm{Fe}^{3+}$ or $\mathrm{Fe}^{0}$, or both.

From the Mössbauer spectra alone it is not possible to decide between these possibilities, but other data from complementary methods may provide additional constraints that allow the type of iron in these components to be assigned. Some of the possibilities that would be consistent with the Mössbauer data are the following

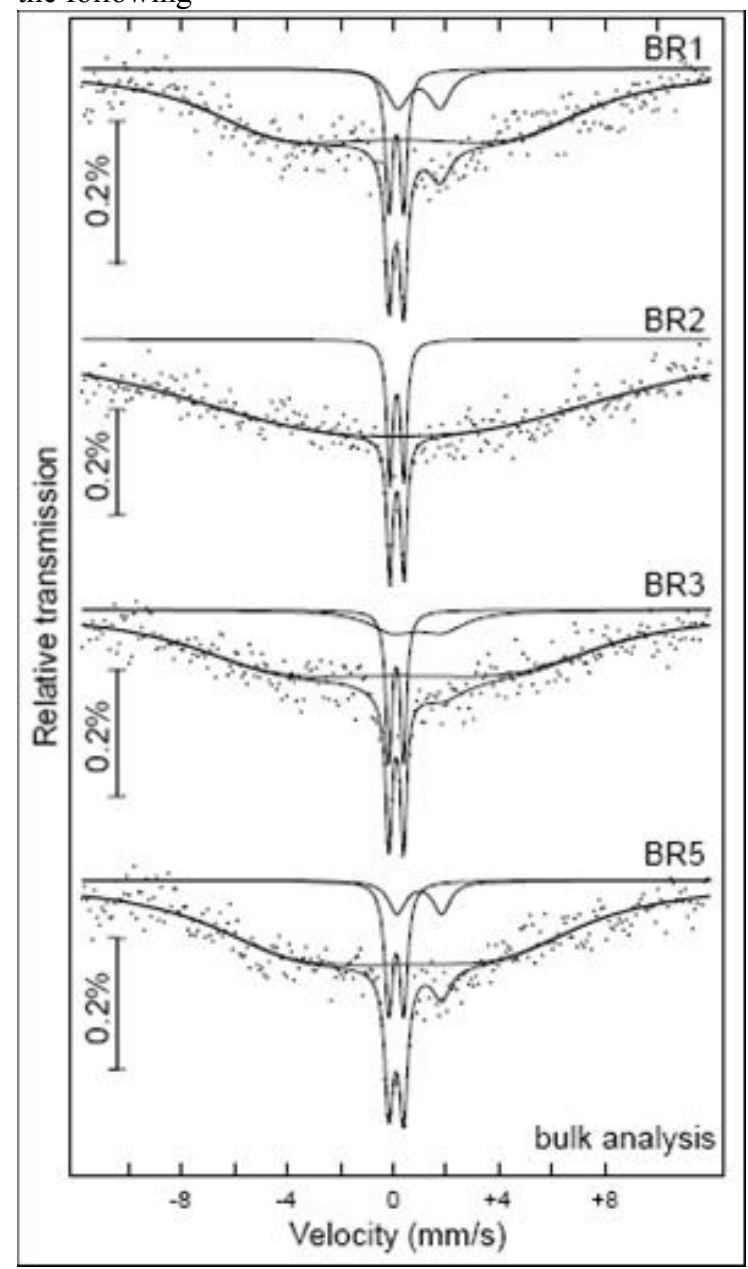

Figure 4. Mössbauer spectra of Brazilizan diamonds (bulk measurements). Samples notation follows Shiryaev et al., (2005).

(1) $\mathrm{Fe}^{3+}$ in low concentrations in a poorly crystallized phase. This could give rise to both the doublet and the sextet:

(2) $\mathrm{Fe}^{3+}$ in a well defined crystalline environment (doublet) and $\mathrm{Fe}^{3+}$ in low concentrations in a poorly crystallised phase (sextet);

(3) $\mathrm{Fe}^{0}$ in an alloy in low concentrations or as fine particles (on the order of $100 \mathrm{~nm}$ ) (doublet + sextet);

(4) various combinations of the above possibilities.

The centre shift of the quadrupole doublet with larger splitting that occurs in some spectra is consistent with $\mathrm{Fe}^{2+}$. The relative areas of components give a first order approximation of the site occupancies; however since the sites may be located in different phases and the relative iron contents of the phases are unknown, the relative areas cannot be used to measure $\mathrm{Fe}^{3+} / \Sigma \mathrm{Fe}$ or phase abundance. If this composition information were available, it would be possible to determine approximate values of these quantities. The spectra of the bulk samples show $\mathrm{Fe}^{2+}$ in all samples with the exception of BR2 (fibrous cube, variety III).

Individual measurements were also made of the core and the rim of the latter sample, and no regions of BR2 
showed the presence of $\mathrm{Fe}^{2+}$ within the detection limit

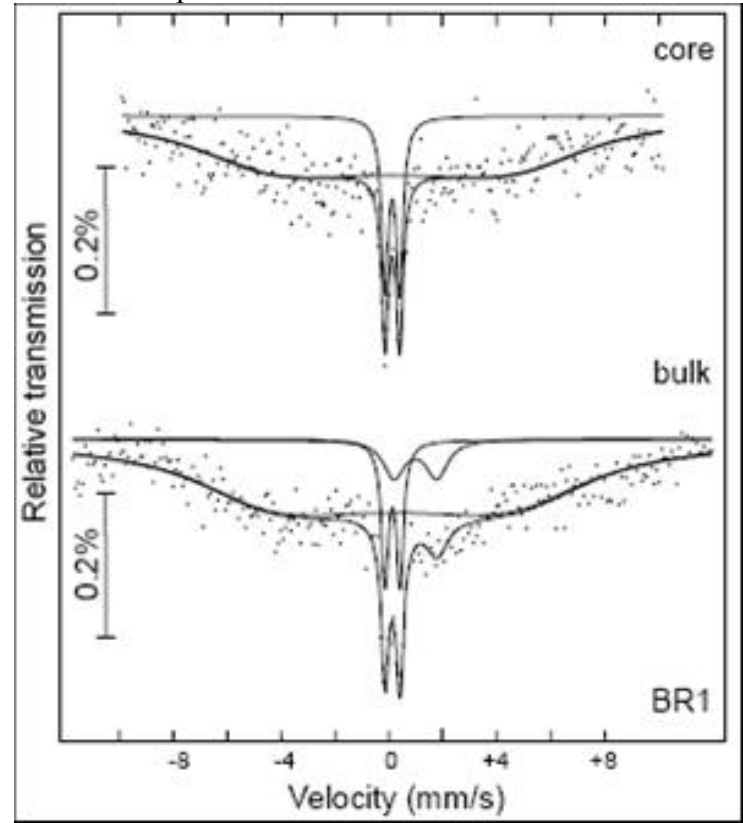

Figure 5. Mössbauer spectra of different parts of the BR1 sample, illustrating differences in Fe redox state for different growth zones.

(estimated to be roughly $3 \%$ of the total area). Coated diamond BR1 did show the presence of $\mathrm{Fe}^{2+}$ in the bulk sample, but no evidence for $\mathrm{Fe}^{2+}$ in the core, implying that $\mathrm{Fe}^{2+}$ is probably concentrated in the rim (Fig. 5). This diamond is highly heterogeneous in $\mathrm{N}$ concentration and $\mathrm{C}$ and $\mathrm{N}$ isotopic composition. Spectroscopic, chemical and isotopic data as well as $\mathrm{CL}$ and $\mathrm{X}$-ray topography make clear that this diamond grew in at least two separate events from different growth mediums. Therefore, the heterogeneity of Fe oxidation state revealed by the current study is not surprising. Sample BR2 contains carbonaceous inclusions and shows a pronounced evolution of $\mathrm{C}$ isotopic composition from center to rim (carbon becomes heavier). This isotopic trend appears to be independent of the current Fe redox measurements.

Acknowledgements: AAS is grateful to the Alexander von Humboldt Foundation and to a Russian President grant MK-147.2007.5 for partial financial support.

\section{References}

Shiryaev A.A., Izraeli E.S., Hauri E.H., Zakharchenko O.D., Navon O. (2005) Chemical, optical and isotopic investigation of fibrous diamonds from Brazil, Russian Geology and Geophysics, 46, 1185-1201

Shiryaev A.A., Ishikawa H., Takegoshi K. (2008) ${ }^{1} \mathrm{H}$ NMR and IR study of hydrogen in fibrous diamonds, Extended Abstracts of XIII All-Russian conference on Thermobarogeochemistry and IV APIFIS symposium, Moscow, www.minsoc.ru/tbg2008/

Wilke M., Farges F., Petit P.-E., Brown Jr. G. E. and Martin F. (2001), Oxidation state and coordination of $\mathrm{Fe}$ in minerals: An Fe K-XANES spectroscopic study. American Mineralogist, 86, 714-730. 